\title{
VORWORT DES DEUTSCHEN BEARBEITERS
}

Die großen Fortschritte in solchen Disziplinen wie Physik, Werkstoffkunde und Technologie haben wesentlich zur raschen Entwicklung der Elektronik beigetragen. Dem Entwickler elektronischer Schaltungen steht so ein weites Spektrum verschiedenartiger leistungsfähiger Bauelemente zur Verfügung. Dabei unterscheiden sich diese Bauelemente mitunter erheblich in den zur Realisierung der vorgesehenen Funktion herangezogenen Wirkungsprinzipien, ihren technischen Parametern und in den spezifischen Einsatzbedingungen. Gleichzeitig wächst mit dem weiteren Vordringen der Elektronik in die verschiedensten Gebiete die Zahl der insgesamt benötigten Bauelemente stark an, so daß durch weitere spezielle Anforderungen an die Technologie neuartige elektronische Bauelemente entstehen. So werden einerseits einzelne Bauelemente eingesetzt, andererseits verlangen bestimmte Anwendungsfälle den Einsatz komplexer integrierter Bauelemente, wie sie die integrierten Schaltungen darstellen. Für den Elektronikingenieur kommt es darauf an, die Wirkungsprinzipien elektronischer Bauelemente und ihre Grundeigenschaften gut zu kennen, um die richtige Auswahl an Bauelementen treffen und mit diesen Bauelementen sachkundig, funktionstüchtige und funktionssichere Schaltungen aufbauen zu können.

Das Buch vermittelt e nen guten Überblick über die große Zahl elektronischer Bauelemente und Grundischaltungen der Digitaltechnik. Ihre Funktionsprinzipien und die bei der Zusammenschaltung zu berücksichtigenden Dimensionierungs-Vorschriften werden erläutert. In der Bearbeitung wurden die Bezeichnungen, Begriffe und Formelzeichen der deutschsprachigen Fachterminologie angeglichen. Zum weiteren Studium dienen einige im hinzugefügten deutschsprachigen Literaturverzeichnis enthaltene Quellen.

August 1972

U. Frühauf 